\title{
O Ensaio de Tiroglobulina com Melhor Sensibilidade Funcional Enquanto os Pacientes Tomam L-T4 Substituirá a Tiroglobulina Estimulada pelo TSH no Seguimento dos Pacientes com Câncer Diferenciado da Tiróide?
}

perspectiva

RUI M.B. MACIEL

Disciplina de Endocrinologia, Departamento de Medicina, Escola Paulista de Medicina/ Universidade Federal de São

Paulo, e Setor de Endocrinologia, Fleury Medicina Diagnóstica, São Paulo, SP.

Recebido em 02/06/07 Aceito em 4/06/07

\section{RESUMO}

O autor apresenta evidências recentes da literatura que mostram que ensaios de tiroglobulina sérica (sTg) com maior sensibilidade funcional apresentam a mesma qualidade que a obtenção da sTg estimulada por rhTSH ou hipotiroidismo, no seguimento de pacientes com câncer diferenciado de tiróide (CDT). Desta forma, propõe modificar a prática recomendada pelas diretrizes de sociedades internacionais para o seguimento desses pacientes (desenvolvidas enquanto os ensaios disponíveis apresentavam sensibilidade de $1 \mathrm{ng} / \mathrm{mL}$ ), substituindo-se a obtenção da sTg estimulada por rhTSH ou hipotiroidismo pelo acompanhamento dos pacientes na vigência da terapia com L-T4 com a medida da sTg desde que se empreguem técnicas com sensibilidade funcional da ordem de 0,1-0,2 ng/mL. (Arq Bras Endocrinol Metab 2007;51/5:862-866)

Descritores: Tiroglobulina; Carcinoma diferenciado de tiróide; Sensibilidade; Sensibilidade funcional; Tiroglobulina estimulada

\section{ABSTRACT}

Will the Thyroglobulin Assay with Lower Functional Sensitivity whilst the Patients are on L-T4 Treatment Replace the TSH-Stimulated Thyroglobulin Assay in the Follow-Up of Patients with Differentiated Thyroid Cancer?

The author reviews the literature on the new assays for serum thyroglobulin (sTg) presenting lower functional sensitivity and demonstrates that its use, whilst the patients are taking L-T4, presents better results than sTg following TSH stimulation in the follow-up of patients with differentiated thyroid carcinoma. Therefore, he suggests a revision on the guidelines for the follow-up of these patients (developed when the available assays present a sensitivity of 1 $\mathrm{ng} / \mathrm{mL}$ ), proposing the use of $\mathrm{sTg}$ assays with functional sensitivity of $0.1-0.2$ $\mathrm{ng} / \mathrm{mL}$ with the patients on L-T4 treatment instead of sTg stimulated by TSH.

(Arq Bras Endocrinol Metab 2007;51/5:862-866)

Keywords: Thyroglobulin; Differentiated thyroid carcinoma; Sensitivity; Functional sensitivity; Stimulated thyroglobulin

\footnotetext{
A S RECOMENDAÇÕES DAS DIRETRIZES recentemente emitidas pelas sociedades norte-americana (ATA) e européia (ETA) de tiróide $(1,2)$, assim como pela Sociedade Brasileira de Endocrinologia e Metabologia (SBEM) - publicada neste volume (3), sobre o seguimento dos pacientes com carcinoma diferenciado da tiróide (CDT), indicam que se devem empregar métodos sensíveis para a deteç̧ão de eventual doença metastática no pescoço ou a distância, pois estas apresentam impacto prognóstico (4). Assim, essas diretrizes determinam que os pacientes com carcinoma diferenciado da tiróide, depois do tratamento inicial, que inclui tiroidectomia total e ablação com iodo radioativo, devem ser seguidos com a dosagem da tiroglobulina sérica $(\mathrm{sTg})$ estimulada pela elevação da tirotrofina (TSH) cerca de 6 a 12 meses após o tratamento inicial (figura 1).
} 


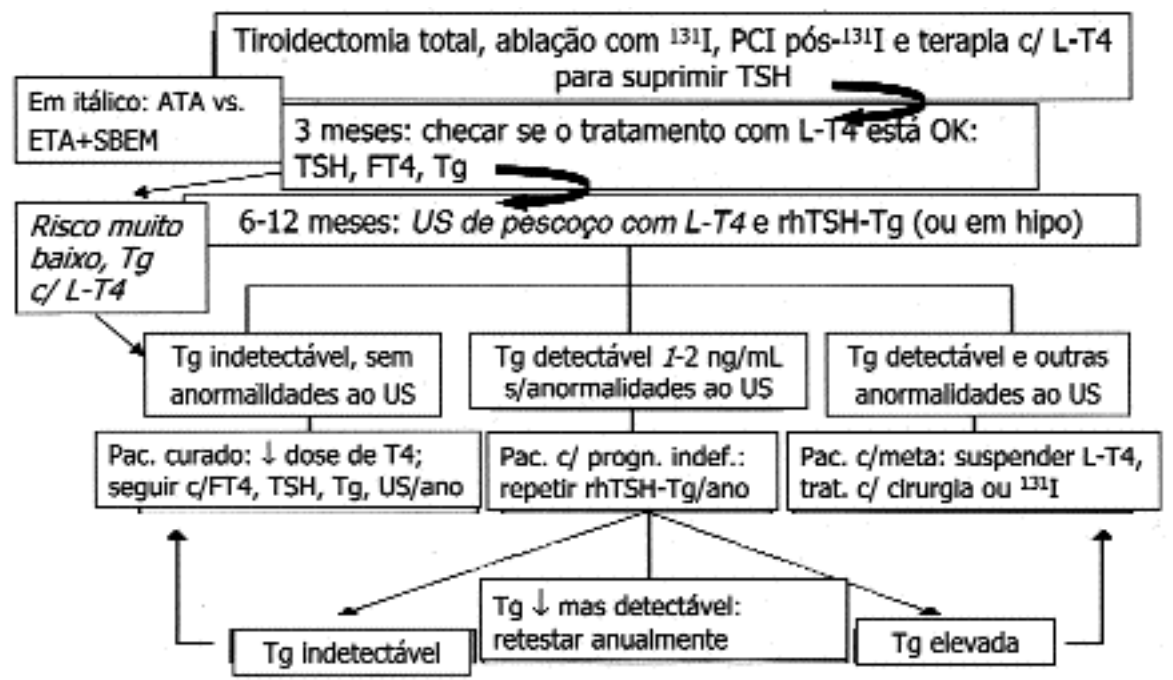

Figura 1. Algoritmos para o seguimento dos pacientes com carcinoma diferenciado da tiróide (CDT) depois do tratamento inicial de acordo com diretrizes da ATA, ETA e SBEM. [Pacini et al., Eur J Endocrinol 2006;154:787; Cooper et al., Thyroid 2006;16:109; Maia et al., Arq Bras Endocrinol Metab 2007;51/5]

É importante ressaltar que essas diretrizes foram baseadas a partir do uso de ensaios de sTg com sensibilidade funcional de $1 \mathrm{ng} / \mathrm{mL}$. Por essas diretrizes, a obtenção da elevação do TSH e, em conseqüência, da sTg estimulada pode ser conseguida seja pelo uso do TSH recombinante (rh-TSH) enquanto o paciente continua recebendo L-T4, seja pela retirada do L-T4 por 4 semanas e conseqüente hipotiroidismo com elevação endógena do TSH (1-3).

A razão desta proposição é decorrente da observação de que a determinação da sTg na vigência de L-T4 pode deixar de detectar pacientes com pequenas quantidades de tumor residual $(1,5,6)$. Assim, sabe-se que aproximadamente $20 \%$ dos pacientes clinicamente livres de doença que apresentam valores de $\mathrm{s} T \mathrm{~g}$ menores do que $\mathrm{l} \mathrm{ng} / \mathrm{mL}$ durante a supressão do TSH com L-T4 mostrarão sTg maior do que $2 \mathrm{ng} / \mathrm{mL}$ depois do estímulo com TSH por rh-TSH ou hipotiroidismo; destes, cerca de $1 / 3$ evidencia alguma forma de tumor residual aos estudos de imagem, enquanto que os $2 / 3$ remanescentes não a demonstram $(5,6)$. Nestes últimos, a $\mathrm{sTg}$ elevada pode ser decorrente quer de restos de tiróide normal, que não foram completamente extirpados pela cirurgia e radioiodo e que se elevam com o TSH, ou então por tumor residual que ainda é pequeno e não pode ser demonstrado aos exames de imagem. É por isso que se recomenda neste sub-grupo de pacientes a obtenção anual da sTg estimulada (1-3) (figura 1).

$\mathrm{Na}$ dependência do valor da sTg encontrado após o estímulo com TSH, dividem-se os pacientes naqueles considerados provavelmente curados (com
sTg indetectável e sem anormalidades à ultra-sonografia cervical), naqueles provavelmente com metástases (com sTg acima de $2 \mathrm{ng} / \mathrm{mL}$ e/ou anormalidades à ultra-sonografia cervical) e naqueles com prognóstico indefinido (com sTg até $2 \mathrm{ng} / \mathrm{mL}$ e sem anormalidades à ultra-sonografia cervical) (figura 1 ).

Entretanto, apesar de indicadas pelas diretrizes, é evidente para os endocrinologistas que lidam com esses pacientes que ambas as técnicas disponíveis para a obtenção da sTg estimulada são problemáticas. Por um lado, o uso do rhTSH, pelo seu elevadíssimo preço, causa impacto nos custos do sistema de saúde, além de eventuais efeitos colaterais indesejáveis (1-3,7); de modo equivalente, a proposta da realização do teste com os pacientes em hipotiroidismo, obtido após a retirada do L-T4, também é nociva, seja pelo quadro agudo de hipotiroidismo, circunstância clínica muito desagradável para os pacientes e que ocasiona muitos dias perdidos de trabalho (7), seja pelos valores elevados de TSH, que podem causar crescimento de eventual massa remanescente de tecido tumoral ou metastático (1-3). Dessa forma, há anos que se postula que o eventual desenvolvimento de um ensaio mais sensível de $\mathrm{s} T \mathrm{~g}$ traria grande benefício ao seguimento desses pacientes, pois poderia tornar desnecessário o estímulo com TSH $(8,9)$.

Recentemente, desenvolveram-se ensaios de sTg com melhor sensibilidade funcional, da ordem de $0,1 \mathrm{ng} / \mathrm{mL}$ ou menos (10-12) e, assim, a literatura passou a examinar a questão se estes ensaios permitiriam a detecção de doença persistente ou recorrente durante a terapêutica com L-T4, sem a necessidade da qualquer estímulo com TSH (13-17). 


\section{RESULTADOS COM OS TRABALHOS UTILIZANDO STg COM MELHOR SENSIBILIDADE FUNCIONAL}

Observações iniciais de Zophel e cols. (10,12-14) compararam a performance de um ensaio do tipo ELISA com sensibilidade funcional de $0,03 \mathrm{ng} / \mathrm{mL}$ com um ensaio imuno-radiométrico (IRMA) convencional e verificaram que a sTg foi detectada pelo ELISA em 106 de 139 (76\%) pacientes seguidos por CDT, e pelo IRMA em apenas 7 dos 139 (5\%). Nenhum paciente apresentava anticorpos anti-tiroglobulina (TgAbs). Desta forma, este estudo indica que o uso de ensaio ultra-sensível de sTg ao invés do ensaio com sensibilidade funcional de $\mathrm{l} \mathrm{ng} / \mathrm{mL}$ apresenta impacto nas diretrizes de seguimento dos pacientes com CDT, uma vez que mostrou, primeiramente, que a sTg é detectável, ao invés de indetectável, na maioria dos pacientes após o tratamento cirúrgico e ablativo com iodo radioativo, e sugere que qualquer elevação da sTg utilizando este tipo de ensaio sensível poderia anunciar precocemente a recorrência da moléstia. A seguir, em outro trabalho, seguiram 126 pacientes com CDT depois do tratamento durante a supressão do TSH; destes, 92 mostraram, a princípio, sTg detectável $(73 \%$, valores de 0,03 a $0,8 \mathrm{ng} / \mathrm{mL})$; durante os 4 anos de seguimento, os níveis de sTg permaneceram inalterados (considerou-se inalterada a medida de sTg que apresentava aumentos menores do que duas vezes o valor basal) em 121/126 (96\%); todos esses pacientes evoluíram bem, sem evidência clínica de metástases. Em 5/126 pacientes, os valores de sTg aumentaram durante o seguimento cerca de duas vezes mais que o valor basal, e em 4 desses detectou-se recorrência da moléstia; no quinto paciente não se evidenciou recorrência, apesar de a sTg continuar subindo e ser estimulada por TSH. Esses resultados indicaram que a medida seriada de sTg realizada com ensaio sensível, sem utilizar o estímulo do TSH, permitiu a detecção de recorrência da doença num período cerca de 6 a 12 meses antes do que se tivesse utilizado o ensaio mais convencional de $\mathrm{sTg}$, com sensibilidade funcional de $\mathrm{l}$ $\mathrm{ng} / \mathrm{mL}(10-14)$.

Posteriormente, Giovanella e cols. analisaram 117 pacientes com CDT tratados com tiroidectomia total e ablação com radioiodo que, após 1 ano do tratamento inicial, submeteram-se à determinação de sTg na vigência de L-T4 empregando ensaio com sensibilidade de $0,2 \mathrm{ng} / \mathrm{mL}$; determinaram-se, também, nestes pacientes, sTg após rhTSH, pesquisa de corpo inteiro com ${ }^{131}$ I e ultra-sonografia cervical. Os resultados indicaram que $10 / 11$ pacientes que apresentaram metástases foram identificados apenas pela determi- nação da sTg sensível e que em apenas 1 caso houve a necessidade da sTg estimulada com TSH. Desta forma, os autores questionam o uso rotineiro de rhTSH, pois houve a necessidade de 104 estimulações com rhTSH para se descobrir apenas 1 caso não detectado pela sTg sensível na vigência de L-T4 (15).

Recentemente, Smallridge e cols. apresentaram a experiência de mais de 4 anos e meio de 2 centros da Mayo Clinic no seguimento de pacientes com CDT utilizando um ensaio de sTg com limite de detecção de $0,1 \mathrm{ng} / \mathrm{mL}$. Assim, de 80 pacientes que apresentavam sTg abaixo de $0,1 \mathrm{ng} / \mathrm{mL}$ na vigência de TSH suprimido, $47(58,8 \%)$ continuaram demonstrando valores indetectáveis após o rhTSH; em 78/80 $(97,5 \%)$ a sTg estimulada foi menor do que $2 \mathrm{ng} / \mathrm{mL}$; em apenas $2 / 80$ dos pacientes, a sTg estimulada após o rhTSH elevou-se para valores de 2,5 e $3 \mathrm{ng} / \mathrm{mL}$. Destes, nenhum apresentou lesão metastática no seguimento. Assim, os autores propõem o uso de sTg sensível na vigência de L-T4, acompanhado da ultrasonografia cervical, como a conduta mais adequada para os pacientes com CDT, ao invés do emprego da determinação da sTg após o estímulo com rhTSH; este seria usado apenas quando o seguimento indicasse a subida nos valores da sTg sensível. Além disso, demonstram que o impacto econômico desta estratégia é significativo, uma vez que a maioria dos pacientes com CDT é de baixo risco e somente $10-20 \%$ apresentam recorrência (16).

Mais recentemente, Schlumberger e cols., num estudo colaborativo e prospectivo que incluiu 944 pacientes, compararam o valor diagnóstico de 7 métodos com sensibilidades funcionais diferentes para a mensuração de sTg no seguimento de pacientes com CDT, tanto durante o tratamento com L-T4 como após a estimulação com TSH. Assim, mediram a sTg em pacientes com CDT que não apresentavam evidência de persistência de doença depois do tratamento inicial (tiroidectomia total e ablação com radioiodo) em duas oportunidades: após 3 meses de tratamento com L-T4 (Tgl) e 9 a 12 meses após, com os pacientes apresentando TSH elevado, seja por hipotiroidismo devido à retirada do L-T4, seja após rhTSH ( $\mathrm{Tg} 2)$. Todos os pacientes eram negativos para TgAbs. Os ensaios de $\mathrm{sTg}$ com sensibilidade funcional de $0,2-0,3 \mathrm{ng} / \mathrm{mL}$ proporcionaram boa sensibilidade e especificidade, fato que levou os autores a concluir que a utilização de ensaios de sTg com menor sensibilidade funcional pode proporcionar uma indicação mais precoce da presença de Tg no soro durante o tratamento com L-T4 e pode ser usado para monitorar a tendência da sTg sem a necessidade da realização de qualquer estímulo do TSH (17). 
Dados preliminares de nosso laboratório também indicam que o emprego de ensaios de sTg com melhor sensibilidade funcional propiciam a detecção precoce de metástases em pacientes com CDT (18).

\section{NOVA PROPOSTA DE DIRETRIZ PARA O SEGUIMENTO DE PACIENTES COM CDT}

Desta forma, tendo em vista estas observações, que demonstram a utilidade do ensaio de sTg com melhor sensibilidade funcional na prática médica, proponho que o seguimento dos pacientes com CDT seja modificado (figura 2). Assim, ao invés de se fazer a sTg estimulada por meio do rhTSH ou hipotiroidismo, acompanharíamos os pacientes com determinações de sTg empregando ensaio com sensibilidade funcional de $0,1-0,2 \mathrm{ng} / \mathrm{mL}$ e ultra-sonografia cervical a cada $6-9$ meses, e a conduta seria determinada pela tendência observada nos valores de sTg. Se estes fossem sempre indetectáveis e os pacientes apresentassem ultra-sonografia cervical sem anormalidades, deveríamos considerar esses pacientes curados e até trocar a dose supressiva de L-T4 pela dose substitutiva, para evitar os efeitos decorrentes do excesso de L-T4. Se os pacientes apresentassem a sTg sensível com tendência de subida, especialmente se acompanhados de alterações à ultra-sonografia cervical, seriam considerados como portadores de metástases e submetidos à cirurgia ou ao tratamento com radioiodo. Se apresentassem sTg com tendência estável ou de queda, mas sem anormalidades à ultra-sonografia cervical, seriam candidatos a seguimento com determinações de $\mathrm{sTg}$ e ultra-sonografia cervical a cada 6 meses/1 ano (figura 2).
Além de simplificar o seguimento dos pacientes com CDT, o impacto econômico desta nova estratégia é significativo, pois a maioria desses pacientes é de baixo risco e apenas 10-20\% apresentam recorrência. O preço de uma única dose de rhTSH para a obtenção da sTg estimulada é de R\$ 6.000,00, enquanto que os valores da sTg sensível estão ao redor de R\$ 130,00, dados que por si só evidenciam o grau de economia de gastos com a proposição do uso do seguimento baseado na sTg com melhor sensibilidade funcional. Além disso, a realização da sTg estimulada por meio do rhTSH exige que o paciente visite o laboratório por 4 dias, comparado às poucas horas necessárias para a dosagem de sTg e para a realização da ultra-sonografia cervical.

\section{O QUE SIGNIFICAM OS VALORES DE STg ENTRE 0,1 E $1 \mathrm{ng} / \mathrm{mL}$ NUM ENSAIO SENSÍVEL DE sTg?}

Sempre que se usa um teste mais sensível no seguimento de pacientes com câncer, há a diminuição correspondente da especificidade, e o médico deve estar atento para fazer a análise criteriosa desses testes. O exemplo do aumento da sensibilidade na determinação do antígeno prostático específico (PSA) no seguimento de pacientes com câncer de próstata traz algumas semelhanças com a melhoria da sensibilidade funcional da $\operatorname{sTg}(19,20)$.

A explicação para a detecção de sTg quando se utiliza a técnica com melhor sensibilidade funcional naqueles indivíduos aparentemente sem doença em seguimento após o tratamento do CDT é devida ao fato de que a tiroidectomia total e a ablação com 131I não conseguem destruir completamente o tecido tiroidiano

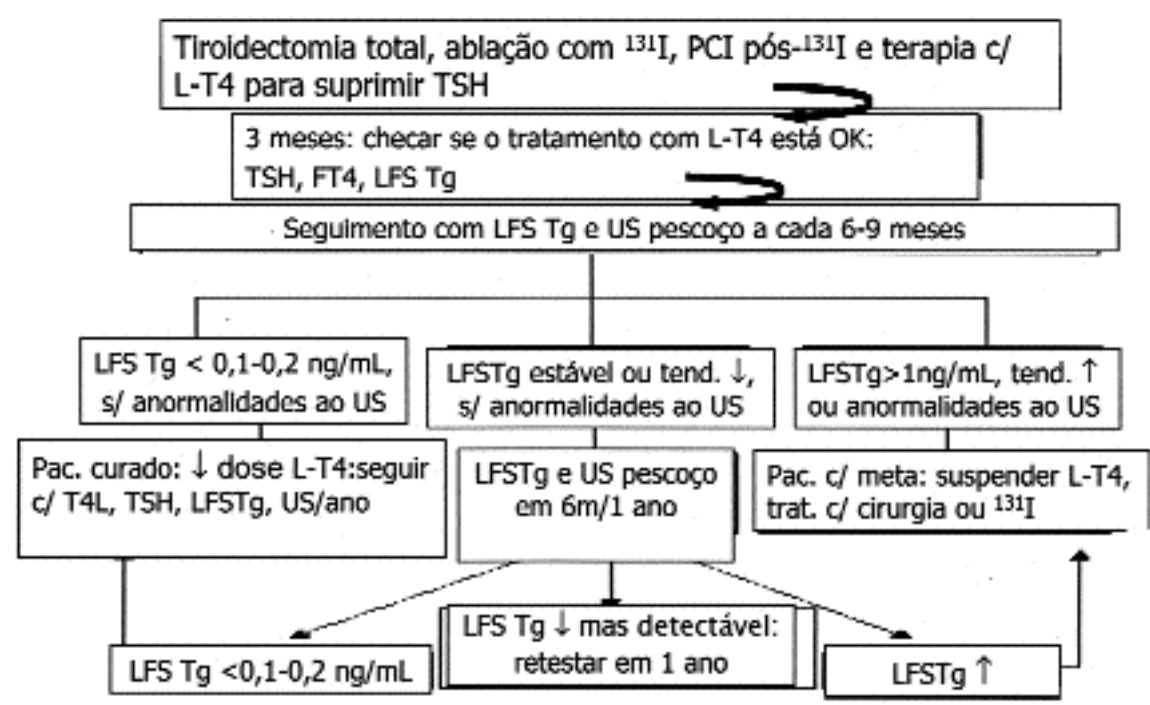

Figura 2. Proposta de algoritmo utilizando sTg com melhor sensibilidade funcional (LFS) para o seguimento de pacientes com CDT após o tratamento inicial. 
normal e, em conseqüência, quantidades mínimas de sTg são dosadas no sangue. À medida, porém, que os efeitos tardios da tiroidectomia, da dose ablativa de radioiodo e, também, da supressão do TSH vão, progressivamente, causando atrofia nos tirócitos, os valores de sTg conseqüentemente diminuem. Assim, por exemplo, Rosário e cols. mostraram que 6/12 pacientes que apresentavam $\mathrm{sTg}$ estimulada $>1 \mathrm{ng} / \mathrm{mL}$ reduziram esses valores para indetectáveis, enquanto que entre os demais 6 pacientes, 5 deles evidenciaram redução superior a $50 \%$ dos valores da sTg estimulada durante um seguimento médio de 24 meses (21), fato anteriormente observado por outros investigadores (22-24).

\section{CONCLUSÕES}

Mudanças de tecnologia podem causar modificações na prática médica, especialmente se são testes mais rápidos, mais baratos e mais eficientes. Parece-me que $\mathrm{o}$ advento dos testes ultra-sensíveis de sTg apresentam estas condições para modificar o seguimento dos pacientes com CDT.

\section{REFERÊNCIAS}

1. Cooper DS, Doherty GM, Haugen BR, Kloos RT, Lee SL, Mandel SJ, et al. Management guidelines for patients with thyroid nodules and differentiated thyroid cancer. Thyroid 2006;16:109-53.

2. Pacini F, Schlumberger $M$, Dralle $H$, Elisei $R$, Smit JWA, Wiersinga W; and the European Thyroid Cancer Task Force. European consensus for the management of patients with differentiated thyroid carcinoma of the follicular epithelium. Eur J Endocrinol 2006;154:787-803.

3. Maia AL, Ward LS, Carvalho GA, Graf H, Maciel RMB, Rosário PW, et al. Nódulos de tiróide e câncer diferenciado de tiróide: consenso brasileiro. Arq Bras Endocrinol Metab 2007;51/5:-.

4. Durante C, Haddy N, Baudin E, Leboulleux S, Hartl D, Travagli $J P$, et al. Long-term outcome of 444 patients with distant metastases from papillary and follicular thyroid carcinoma: benefits and limits of radioiodine therapy. J Clin Endocrinol Metab 2006;91:2892-9.

5. Schlumberger M, Berg G, Cohen O, Duntas L, Jarnar F, Jarzab B, et al. Follow-up of low risk patients with differentiated thyroid carcinoma: an European perspective. Eur J Endocrinol 2004;150:105-12.

6. Mazzaferri EL, Robbins RJ, Braverman LE, Pacini F, Haugen BR, Wartofsky L, et al. Author's response: A consensus report of the role of serum thyroglobulin as a monitoring method for low-risk patients with papillary thyroid carcinoma. J Clin Endocrinol Metab 2003;88:4508-9.

7. Duntas LH, Biondi B. Short-term hypothyroidism after L-T4 withdrawal in patients with differentiated thyroid cancer: clinical and quality of life consequences. Eur $\mathbf{J}$ Endocrinolol 2007; 156:13-9.

8. Mazzaferri EL, Robbins RJ, Spencer CA, Braverman LE, Pacini $F$, Wartofsky $L$, et al. A consensus report of the role of serum thyroglobulin as a monitoring method for low-risk patients with papillary thyroid carcinoma. J Clin Endocrinol Metab 2003;88:1433-41.
9. Spencer CA, Bergoglio LM, Kazarozyan M, Fatemi S, LoPresti JS. Clinical impact of thyroglobulin $(\mathrm{Tg})$ and $\mathrm{Tg}$ antibody method differences on the management of patients with differentiated thyroid carcinomas. J Clin Endocrinol Metab 2005;90:5556-75.

10. Wunderlich G, Zophel K, Crook L, Smith S, Smith BR, Franke WG. A high-sensitivity enzyme-linked immunosorbent assay for serum thyroglobulin. Thyroid 2001;11:819-24.

11. Mongenthaler NG, Froelich J, Rendl J, Willnich M, Alonso C, Bergmann A, et al. Technical evaluation of a new immunoradiometric and an immunoluminometric assay for thyroglobulin. Clin Chem 2002:48:1077-83.

12. Zophel K, Wunderlich G, Smith BR. Serum thyroglobulin measurements with a high-sensitivity assay enzyme-linked immunosorbent assay: is there a clinical benefit in patients with differentiated thyroid carcinoma? Thyroid 2003;13:861-5.

13. Zophel K, Wunderlich G, Franke WG. Initial experiences with a highly sensitive enzyme-linked-immuno-sorbent assay (ELISA) for measurement of thyroglobulin in patients with differentiated thyroid carcinoma. J Lab Med 2002;26:425-33.

14. Zophel K, Wunderlich G, Kotzerke J. A highly sensitive thyroglobulin assay has superior diagnostic sensitivity for recurrence of differentiated thyroid cancer in patients undergoing TSH suppression. J Nucl Med 2006;47:552-3.

15. Giovanella L, Ceriani L, Ghelfo A, Keller F, Sacchi A, Maffioli $M$, et al. Thyroglobulin assay during thyroxine treatment in low-risk differentiated thyroid cancer management: comparison with recombinant assay and imaging procedures. Clin Chem Lab Med 2006; 44:648-52.

16. Smallridge RC, Meek SE, Morgan MA, Gates GS, Fox TP, Grebe $S$, et al. Monitoring thyroglobulin in a sensitive immunoassay has comparable sensitivity to recombinant human TSH-stimulated thyroglobulin in follow-up of thyroid cancer patients. J Clin Endocrinol Metab 2007;92:82-7.

17. Schlumberger M, Hitzel A, Toubert ME, Corone C, Troalen F, Schlageter $\mathrm{MH}$, et al. Comparison of seven serum thyroglobulin assays in the follow-up of papillary and follicular thyroid cancer patients. J Clin Endocrinol Metab 2007;92:[Epub ahead of print].

18. Biscolla RP, Ikejiri ES, Mamone MC, Nakabashi CC, Andrade VP, Kasamatsu TS, et al. Diagnóstico de metástases de carcinoma papilífero de tiróide através da dosagem de tiroglobulina no líquido obtido da lavagem da agulha utilizada na punção aspirativa. Arq Bras Endocrinol Metab 2007;51/3:419-25.

19. Kobayashi T, Goto R, Ito K, Mitsumori K. Prostate cancer screening strategies with re-screening interval determined by individual baseline prostate-specific antigen values are costeffective. Eur J Surg Oncol 2007; [Epub ahead of print].

20. Ankerst DP, Thompson IM. Sensitivity and specificity of prostate-specific antigen for prostate cancer detection with high rates of biopsy verification. Arch Ital Urol Androl 2006;78:125-9.

21. Rosário $\mathrm{P}$, Borges $\mathrm{M}$, Reis J, Alves MF. Effect of suppressive therapy with levothyroxine on the reduction of serum thyroglobulin after total thyroidectomy. Thyroid 2006;16:199-200.

22. Rosário PW, Borges MA, Fagundes TA, Franco AC, Purisch S. Is stimulation of thyroglobulin useful in low-risk patients with thyroid carcinoma and undetectable $\mathrm{Tg}$ on thyroxine and negative neck ultrasound? Clin Endocrinol 2005;62:121-5.

23. Cailleux AF, Baudin E, Travagli JP, Ricard M, Schlumberger $M$. Is diagnostic ${ }^{131}$ I scanning useful after total thyroid ablation for differentiated thyroid cancer? J Clin Endocrinol Metab 2000;85:175-8.

24. Torlontano M, Attard M, Croceti U, Tumino S, Bruno R, Costante $\mathrm{G}$, et al. Follow-up of low risk patients with papillary thyroid cancer: role of neck ultrasonography in detecting lymph node metastases. J Clin Endocrinol Metab 2004;89:3402-7.

Endereço para correspondência:

Rui M.B. Maciel

Laboratório de Endocrinologia Molecular

Rua Pedro de Toledo 781, $12^{\circ}$ andar

04039-032 São Paulo, SP

E-mail: rmbmaciel-endo@pesquisa.epm.br 